\title{
Comparative cost-effectiveness of immunoassays and FLOTAC for diagnosing Giardia spp. infection in dogs
}

\author{
Paola Pepe ${ }^{1}$, Davide lanniello ${ }^{1}$, Leucio Camara Alves ${ }^{2 *}$, Maria Elena Morgoglione ${ }^{1}$, Maria Paola Maurelli ${ }^{1}$, \\ Antonio Bosco ${ }^{1}$, Giuseppe Cringoli ${ }^{1}$ and Laura Rinaldi ${ }^{1}$
}

\begin{abstract}
Background: Giardia spp. is a protozoan pathogen and is the most common enteric parasite of domestic animals and humans. Assays for detecting infection in fecal samples using direct or indirect examinations are important tools for diagnosing the disease. The objective of the present study was to compare the cost-effectiveness of immunoassays and FLOTAC technique for diagnosing Giardia spp. infection in dogs.

Results: Fecal samples from 80 positive stray dogs were tested for the presence of copro-antigens of Giardia spp. using the direct immunofluorescence assay (IFA), a rapid enzyme-linked immunosorbent assay (ELISA) and the FLOTAC double technique. All methods were performed in accordance with the instructions reported in the original description for each technique. The results showed that ELISA can be run in less time than IFA and almost at the same time of the FLOTAC technique. Among the tests used in this study, FLOTAC had the lowest cost per correct diagnosis, compared with immunoassays.

Conclusions: The results from this cost-effectiveness analysis, in combination with the sensitivity and specificity of the FLOTAC technique, suggest that the FLOTAC technique can be use in the routine diagnosis of Giardia spp. infection in dogs.
\end{abstract}

Keywords: Giardia spp., Immunoassays, Diagnosis, FLOTAC

\section{Background}

Among protozoal infections, giardiasis is the most common disease in a wide variety of animals, including humans [1]. Once a person or animal has become infected with Giardia spp., zoonotic transmission cycles may occur [2]. In fact, new evidence has shown that there is a strict genetic relationship between some G. duodenalis genotypes isolated from infected humans and dogs [3]. In particular, assemblages A (subtypes I and II) and B (subtypes I and IV) have been associated with human infections [4], but are also found in a number of other mammalian hosts [5], and assemblage $C$ from dogs was

\footnotetext{
*Correspondence: leucioalves@gmail.com

${ }^{2}$ Department of Veterinary Medicine, Federal Rural University of Pernambuco, R. Manuel de Medeiros s/n, Dois Irmãos, Recife, PE 52171-900, Brazil

Full list of author information is available at the end of the article
}

found in humans in Europe [6], thus suggesting the possibility of interspecies transmission [7].

Giardia infection in dogs is an important disease in veterinary medicine [8] and infected animals show clinical signs of disease two to three weeks after infection [9], characterized by diarrhea, vomiting, weight loss and lethargy. However, some animals do not present any clinical signs [10]. Surveys on a variety of canine populations have revealed a prevalence of Giardia infection ranging from $10 \%$ among well-cared-for dogs to $36-50 \%$ among puppies and up to $100 \%$ among kennel dogs, which are at highest risk of disease transmission [11]. Many factors appear to affect the prevalence of the infection including the animal's characteristics (age, living conditions, animal density, nutritional status and immune status) and the diagnostic test used [12]. 
Assays for detecting infection in fecal samples using direct or indirect examinations are important tools for diagnosing the disease [13-15]. In general, the diagnosis is based on the detection of Giardia cysts (and occasionally trophozoites) in the feces of infected dogs [16]. The traditional approaches, such as use of fecal smears and flotation in tubes, have significant limitations due to the small size of the cysts. Moreover, shedding of cysts is intermittent, even in chronically infected individuals, thus requiring multi-day fecal examination [17]. Therefore, more sensitive diagnostic immunoassays such as the immunofluorescence assay (IFA; regarded as the "gold standard"), immunochromatography and the enzyme-linked immunosorbent assay (ELISA) [18] have been recognized as important tools for detecting Giardia spp. in fecal samples from dogs.

A new technique known as FLOTAC has been developed and proposed for diagnosing enteric parasites in animals and humans. In several studies, it has been shown to have high sensitivity, specificity and accuracy [19]. The aim of this study was to compare the costeffectiveness of immunoassays and FLOTAC technique for diagnosing Giardia spp. infection in dogs.

\section{Results}

Among the 80 samples examined, all (100\%) were found to be positive by the FLOTAC test. The only test that revealed a negative sample was ELISA.

The costs of all the kits were ascertained based on an internet survey of the commercial kits available for diagnosing Giardia spp. (Table 1). The time taken to analyze the samples using each of the techniques and the sensitivity and specificity of each diagnostic test kit for Giardia spp. are shown in Tables 2 and 3, respectively.

Comparing the sensitivity and specificity of these tests, FLOTAC and IFA have the same capability to diagnose Giardia spp. infection in dogs but the FLOTAC technique showed higher sensitivity than ELISA. The Kappa test showed a good and a very good agreement of 1.00 (IFA/FLOTAC) and 0.98 (IFA/ELISA), respectively.

Table 1 Direct costs of diagnosis kits of Giardia infection based on an internet survey of commercial kits

\begin{tabular}{llll}
\hline Test & Mean (US\$) & Minimum (US\$) & Maximum (US\$) \\
\hline ELISA & 11.4 & 8.71 & 16.3 \\
IFA & 9.8 & 7.20 & 14.6 \\
FLOTAC & 1 & 0.50 & 1.5 \\
\hline
\end{tabular}

\section{Discussion}

The coproparasitological diagnostic tests for Giardia spp. in dogs that have been used include direct smears, fecal flotation, centrifugal fecal flotation, IFA, ELISA and polymerase chain reaction (PCR) assay. These tests can be used either alone or in combinations in order to improve the sensitivity [20]. Moreover, it has been reported that to make a true diagnosis of Giardia spp. infection in dogs, immunoassays need to be used because the sensitivity and specificity of these tests are higher [17]. Onestep ELISA and immunofluorescence assays have been recognized as important tools for detecting Giardia spp. in fecal samples from dogs $[17,21-23]$.

Several studies comparing diagnostic tests for Giardia spp. infection in dogs have shown that parasitological tests and immunoassays have similar performance [24] and that they need to be used together [25]. On the other hand, almost all studies have shown that immunoassays were more sensitive and that they improved the accuracy of diagnosing Giardia spp. infection in dogs [17, 21-23, 26-28].

IFA is the serological test that is most used for diagnosing Giardia spp. infection, given that it is regarded as the gold-standard test. ELISA is also widely used, not only because it is a highly sensitive and specific test, but also because it is very easy to use. Since immunoassays detect antigens, it can be expected that both ELISA and IFA would detect more dogs as positive than would tests based on cysts, such as the FLOTAC technique.

Table 2 Giardia infection according to target of test, time and cost by sample

\begin{tabular}{llll}
\hline Test & Target & Result (min) & Cost/sample \\
\hline ELISA & Antigen & $11-12$ & +++ \\
IFA & Antigen & $40-50$ & ++ \\
FLOTAC & Parasite & $12-15$ & + \\
\hline
\end{tabular}

Note: One sample is required for all tests

Table 3 Evaluation of immunoassay tests and FLOTAC technique compared to the immunofluorescence antibody test as a gold test in diagnosis of Giardia spp. infection in dogs

\begin{tabular}{lll}
\hline Parameter (\%)/technique & ELISA & FLOTAC \\
\hline Sensitivity & 98.75 & 100 \\
Specificity & 100 & 100 \\
True prevalence & 100 & 100 \\
Estimated prevalence & 98.00 & 100 \\
Predictive value (+) & 100 & 100 \\
Predictive value (-) & 0 & 0 \\
Accuracy & 98.75 & 100 \\
Incorrect classification & 1.25 & 0 \\
\hline
\end{tabular}


However, in the present study, it was observed that the FLOTAC technique showed the same sensitivity and specificity as IFA and a higher sensitivity than ELISA.

Dog owners generally associate giardiasis when their pets presenting the symptom of diarrhea. However, some animals remain asymptomatic [16] and sometimes they are erroneously treated. These animals present lower numbers of cysts in stool samples and false-negative test results may occur. In this regard, antigen tests are not indicated for the follow-up of patients with persistent symptoms after being treated for giardiasis, because the test sensitivity is compromised [29].

For detection of intestinal protozoa, the FLOTAC technique has been reported as a promising test in comparison with other parasitological techniques [30]. Furthermore, Speich et al. [31] made a comparative cost assessment of the FLOTAC and Kato-Katz techniques for diagnosing soil-transmitted helminths, and they found that the cost of the FLOTAC technique was higher than the cost of Kato-Katz, when salaries and costs due to materials and infrastructure were included. However, to our knowledge, the present study is the first to compare FLOTAC with immunoassays.

To make a diagnosis of dog's giardiasis, the laboratories should have the necessary equipment for the accomplishment of the tests, particularly for immunoassays. All three techniques, IFA and ELISA and FLOTAC, can be performed at an unsophisticated laboratory, but FLOTAC is a diagnostic tool that is easy to apply for routine diagnosis of Giardia spp. infection in dogs.

Analysis on the time taken and the samples required for making the diagnosis using each of the techniques showed that ELISA could be run in a shorter time than IFA and that this time was closer to that required for the FLOTAC technique (Table 2). The time that has elapsed between the onset of clinical signs and making the diagnosis of giardiasis in dogs is an important point because some animals show severe diarrhea that may be fatal if left untreated.

Among the tests used in this study, FLOTAC had the lowest cost per correct diagnosis, in comparison with the immunoassays (Table 1).

In this study there was very good agreement between results obtained with IFA and FLOTAC. The discordance between IFA and ELISA assay can be explained by the detection limits for this test, which detects cyst wall proteins [32].

\section{Conclusions}

The results from this cost-effectiveness analysis, in combination with the sensitivity and specificity of the FLOTAC technique, suggest that the FLOTAC technique can be used in making routine diagnoses of Giardia spp. infection in dogs.

\section{Methods}

The objective of the present study was to compare the cost-effectiveness of immunoassays and the FLOTAC technique for diagnosing Giardia spp. infection in dogs. A total of 80 positive fecal samples according to the goldstandard IFA test, were included in this study. All samples were from stray dogs living in the city of Naples (Campania region, southern Italy) that had been brought to the veterinary Hospital of the School of Veterinary Medicine. The technicians were blinded to patient history and results of tests.

Three methods were used: the IFA test using a MeriFluor $^{\circledR}$ Cryptosporidium/Giardia, (Meridian Bioscience Diagnostic, Cincinnati, OH, USA), a rapid ELISA using the IDEXX SNAP ${ }^{\circledR}$ test (Idexx Laboratories Inc., Schiphol-Rijk, Netherlands), and the FLOTAC double technique [19] in which zinc sulfate (specific gravity = 1.350) was used as the flotation solution. Magnifications of $100 \times$ and $400 \times$ were used to identify protozoan cysts. The results were expressed as the arithmetic mean of the number of cysts per gram (CPG) of feces. In order to evaluate cost-effectiveness, the IFA test was used as the gold-standard test.

In using IFA, the numbers of Giardia spp. cysts found were ranked into the following three levels: 1 (1 cyst); 2 (1-2 cysts); and 3 (3-4 cysts) per reading area.

All methods were performed in accordance with the manufacturer's instructions. The sensitivity, specificity, positive predictive value $(+\mathrm{PV})$, negative predictive value (-PV), accuracy, true estimated prevalence and incorrect classification were determined in comparison to the IFA technique as the gold standard. The InStat software 3.01 (GraphPad Software, Inc., San Diego, California, USA) was used to calculate all parameters. To assess the costeffectiveness of the immunoassays and the FLOTAC it was considered that laboratories would have all the necessary equipment to undertake the tests. To calculate a measure of agreement between IFA, ELISA and FLOTAC, the results were assessed using Cohn's Kappa coefficient with $95 \%$ confidence interval.

Abbreviations

IFA: immunofluorescence antibody test; ELISA: Enzyme-linked immunosorbent assay; CPG: cysts per gram.

Acknowledgements

Not applicable.

Funding

Funding for this study and the publication of the results was provided by the authors. 


\section{Availability of data and materials}

The datasets supporting the conclusions of this article are included within the article.

\section{Authors' contributions}

PP, DI, MEM, MPM and AB carried out analysis, interpreted data and helped to draft manuscript. PP and LCA drafted the manuscript. GC and LR conceived and coordinated the study. All authors read and approved the final manuscript.

\section{Ethics approval and consent to participate}

Not applicable.

\section{Consent for publication}

Not applicable.

\section{Competing interests}

The authors declare that they have no competing interests.

\section{Publisher's Note}

Springer Nature remains neutral with regard to jurisdictional claims in published maps and institutional affiliations.

\section{Author details}

${ }^{1}$ Department of Veterinary Medicine and Animal Production, University of Naples Federico II, CREMOPAR, Campania Region, Eboli, Italy. ${ }^{2}$ Department of Veterinary Medicine, Federal Rural University of Pernambuco, R. Manuel de Medeiros s/n, Dois Irmãos, Recife, PE 52171-900, Brazil.

Received: 14 November 2018 Accepted: 29 March 2019

Published online: 08 April 2019

\section{References}

1. Robertson LJ. Giardia duodenalis. In: Percival SL, Yates MV, Williams DW, Chalmers RM, Gray NF, editors. Microbiology of waterborne diseases. 2nd ed. London: Elsevier; 2014. p. 375-405.

2. Esch KJ. Transmission and epidemiology of zoonotic protozoal diseases of companion animals. Clin Microbiol Rev. 2013;26:58-85.

3. Marangi M, Berrilli F, Otranto D, Giangaspero A. Genotyping of Giardia duodenalis among children and dogs in a closed socially deprived community from Italy. Zoonoses Public Health. 2010;57:54-8.

4. Zheng G, Alsarakibi M, Liu Y, et al. Genotyping of Giardia duodenalis isolates from dogs in Guangdong, China based on multi-locus sequence. Korean J Parasitol. 2014:52:299-304.

5. Vanni I, Cacciò SM, Van Lith L, Lebbad M, Svärd SG, Pozio E, et al. Detection of Giardia duodenalis assemblages A and B in human feces by simple, assemblage-specific PCR assays. PLoS Negl Trop Dis. 2012;6:e1776.

6. Štrkolcová G, Mad’ar M, Hinney B, Goldová M, Mojžišová J, Halánová M. Dog's genotype of Giardia duodenalis in human: first evidence in Europe. Acta Parasitol. 2015;60:796-9.

7. Capelli G, Regalbono FA, lorio R, Pietrobelli M, Paoletti B, Giangaspero A. Giardia species and other intestinal parasites in dogs in north-east and central Italy. Vet Rec. 2006;159:422-4.

8. Bouzid M, Halai K, Jeffreys D, Hunte PR. The prevalence of Giardia infection in dogs and cats, a systematic review and meta-analysis of prevalence studies from stool samples. Vet Parasitol. 2015;30:181-202.

9. Serradell MC, Saura LA, Rupil P, Gargantini R, Faya MI, Furlan PJ, et al. Vaccination of domestic animals with a novel oral vaccine prevents Giardia infections, alleviates signs of giardiasis and reduces transmission to humans. NPJ Vaccines. 2016;1:16018.

10. Volkmann MJM, Steiner GT, Fosgate J, Zentek S, Kohn B. Chronic diarrhea in dogs - retrospective study in 136 cases. J Vet Intern Med. 2017;31:1043-55.

11. Uchôa FFM, Sudré AP, Campos SDE, Almosny NRP. Assessment of the diagnostic performance of four methods for the detection of Giardia duodenalis in fecal samples from human, canine and feline carriers. J Microbiol Methods. 2018;145:73-8.
12. Thompson RCA, O'Handley R, Palmer CS. The public health and clinical significance of Giardia and Cryptosporidium in domestic animals. Vet J. 2007:177:18-25.

13. Al-Saeed AT, Issa SH. Detection of Giardia lamblia antigen in stool specimens using enzyme-linked immunosorbent assay. East Mediterr Health J. 2010;16:362-4.

14. Weitzel T, Dittrich S, Möhl I, Adusu E, JelinekT. Evaluation of seven commercial antigen detection tests for Giardia and Cryptosporidium in stool samples. Clin Microbiol Infect. 2006;12:656-9.

15. Salman Y. Efficacy of some laboratory methods in detecting Giardia lamblia and Cryptosporidium parvum in stool samples. KUJSS. 2014;9:7-17.

16. Olson ME, Leonard NJ, Strout J. Prevalence and diagnosis of Giardia infection in dogs and cats using a fecal antigen test and fecal smear. Can Vet J. 2010:51:640-2.

17. Rishniw M, Liotta J, Bellosa M, Bowman D, Simpson KW. Comparison of 4 Giardia diagnostic tests in diagnosis of naturally acquired canine chronic subclinical giardiasis. Vet Intern Med. 2010;24:293-7.

18. Jahan N, Khatoon R, Ahmad SA. Comparison of microscopy and enzyme linked immunosorbent assay for diagnosis of Giardia lamblia in human faecal specimens. J Clin Diagn Res. 2014;8:DC04-6.

19. Cringoli G, Rinaldi L, Maurelli MP, Utzinger J. FLOTAC: new multivalent technique for qualitative and quantitative copromicroscopic diagnosis of parasites in animals and humans. Nat Protoc. 2010;5:503-15.

20. Tangtrongsup S, Scorza V. Update on the diagnosis and management of Giardia spp. infections in dogs and cats. Top Companion Anim Med. 2010;25:155-62.

21. Cerak VY, Bauer C. Comparison of conventional coproscopical methods and commercial coproantigen ELISA kits for the detection of Giardia and Cryptosporidium infections in dogs and cats. Berl Münch Tierärztl Wochenschr. 2004;117:410-3.

22. Gundłach JL, Sadzikowski AB, Stepień-Rukasz H, Studzińska MB, Tomczuk K. Comparison of some serological methods and coproscopic examinations for diagnosis of Giardia spp. invasion in dogs. Pol J Vet Sci. 2005;8:137-40

23. Dryden MW, Payne PA, Smith V. Accurate diagnosis of Giardia spp. and proper fecal examination procedures. Vet Ther. 2006;7:4-14.

24. Uehlinger F, Naqvi SA, Greenwood SJ, Mcclure Trenton J, Conboy G, et al. Comparison of five diagnostic tests for Giardia duodenalis in fecal samples from young dogs. Vet Parasitol. 2017;15:91-6.

25. Costa M, Clarke C, Mitchell S, Papasouliotis K. Diagnostic accuracy of two point-of-care kits for the diagnosis of Giardia species infection in dogs. J Small Anim Pract. 2016:57:318.

26. Zimmer JF, Burrington DB. Comparison of four techniques of fecal examinations for detecting canine giardiasis. J Am Anim Hosp Assoc. 1986;22:161-7

27. Decock C, Cadiergues MC, Larcher M, Vermot S, Franc M. Comparison of two techniques for diagnosis of giardiasis in dogs. Parasite. 2003;10:69-72.

28. Geurden T, Berkvens D, Casaert S, Vercruysse J, Claerebout E. A Bayesian evaluation of three diagnostic assays for the detection of Giardia duodenalis in symptomatic and asymptomatic dogs. Vet Parasitol. 2008;157:14-20.

29. Strand EA, Robertson LJ, Hanevik K, Alvsvåg JO, Mørch K, Langeland N. Sensitivity of a Giardia antigen test in persistent giardiasis following an extensive outbreak. Clin Microbiol Infect. 2008;14:1069-71.

30. Becker SL, Lohourignon KL, Speich B, Rinaldi L, Knopp S, N'goran KE, et al. Comparison of the Flotac-400 dual technique and the formalin-ether concentration technique for diagnosis of human intestinal protozoon infection. J Clin Microbiol. 2011;49:2183-90.

31. Speich B, Knopp S, Mohammed KA, Khamis IS, Rinaldi L, Cringoli G, et al. Comparative cost assessment of the Kato-Katz and FLOTAC techniques for soil-transmitted helminth diagnosis in epidemiological surveys. Parasit Vectors. 2010;3:71.

32. Uiterwijk M, Nijsse R, Kooyman F, Wagenaar JA, Mughini-Gras L, Koop G, Ploeger HW. Comparing four diagnostic tests for Giardia duodenalis in dogs using latent class analysis. Parasit Vectors. 2018;11:439. 